\title{
Quasinormal modes of the BTZ black hole are generated by surface waves supported by its boundary at infinity
}

\author{
Yves Décanin $⿴ 囗 十$ and Antoine Folacci \\ UMR CNRS 6134 SPE, Equipe Physique Semi-Classique (et) de la Matière Condensée \\ Université de Corse, Faculté des Sciences, BP 52, 20250 Corte, France
}

(Dated: November 7, 2018)

\begin{abstract}
We develop the complex angular momentum method in the context of the BTZ black hole physics. This is achieved by extending a formalism introduced a long time ago by Arnold Sommerfeld, which allows us to define and use the Regge pole concept in a framework where the notion of an $S$ matrix does not exist. The Regge poles of the BTZ black hole are exactly obtained and from the associated Regge trajectories we determine its quasinormal mode complex frequencies. Furthermore, our approach permits us to physically interpret them: they appear as Breit-Wigner-type resonances generated by surface waves supported by the black hole boundary at infinity which acts as a photon sphere.

PACS numbers: 04.70.-s
\end{abstract}

\section{INTRODUCTION}

By using complex angular momentum (CAM) techniques, we showed some years ago that the quasinormal mode (QNM) complex frequencies of the Schwarzschild black hole of mass $M$ are Breit-Wigner-type resonances generated by a family of "surface waves" lying on its photon sphere at $r=3 M[1]$. More precisely, by noting that each surface wave is associated with a Regge pole of the $S$ matrix of the Schwarzschild black hole [1, 2, 3], we have been able to construct the spectrum of the QNM complex frequencies from the Regge trajectories, i.e., from the curves traced out in the CAM plane by the Regge poles as a function of the frequency. In this way, we have established, on a "rigorous" basis, an appealing and physically intuitive interpretation of the Schwarzschild black hole QNMs suggested by Goebel in 1972 [4], i.e., that they could be interpreted in terms of gravitational waves in spiral orbits close to the unstable circular photon orbit at $r=3 M$ which decay by radiating away energy (see also Refs. [5, [6, 7, 8] for alternative implementations of the Goebel interpretation).

Now, it is natural to use the CAM approach in order to physically understand the resonant aspects of more general black holes. We believe that, mutatis mutandis, it should be fairly easy to generalize the CAM analysis developed in Refs. [1, 2, 3] for all the asymptotically flat black holes with a photon sphere. By contrast, for black holes immersed in a non-asymptotically flat background, i.e., in a framework where the notion of an $S$ matrix does not exist, we can expect to encounter new difficulties. However, because such black holes play a central

\footnotetext{
*Electronic address: decanini@univ-corse.fr

${ }^{\dagger}$ Electronic address: folacci@univ-corse.fr
}

role in the context of superstring theory and quantum gravity, their CAM analysis seems to be an interesting and important task which could, in particular, shed light on AdS/CFT correspondence and holography from a new point of view.

In the present paper, our aim is more modest even if we try to make some steps in this direction. We shall consider the most simple black hole immersed in an asymptotically anti-de Sitter space-time, namely the $(2+1)$ dimensional Bañados-Teitelboim-Zanelli (BTZ) black hole 9]. It seems to us very interesting in order to test our approach because, in that particular space-time, the wave equation can be solved exactly [10, 11, 12, 13]. We shall revisit the QNM problem for this black hole from the point of view of the CAM approach in order to analyze it semiclassically, i.e., in term of surface waves. In Sec. II, we shall define the Regge poles and the associated Regge modes for a massless scalar field defined on the BTZ black hole by extending a formalism introduced a long time ago by Sommerfeld [14] as an alternative to the usual Watson approach of scattering [15]. It permits us to use the Regge pole concept in a framework where the notion of an $S$ matrix does not exist. The Regge poles and the Regge modes of the BTZ black hole are exactly obtained and physically interpreted in terms of surface waves supported by its boundary, this boundary furthermore playing the role of a photon sphere. In Sec. III, we shall first construct from the Regge modes the diffractive part of the Feynman propagator associated with the scalar field. We shall then show that the poles of its temporal Fourier transform are the QNM complex frequencies of the BTZ black hole and prove that they are generated by the surface waves supported by the black hole boundary. In a short conclusion, we shall make some remarks concerning the AdS/CFT correspondence and the possibility to consider black hole photon spheres as holographic screens. 


\section{REGGE MODES OF THE BTZ BLACK HOLE}

\section{A. Quasinormal modes and Regge modes of the BTZ black hole}

The metric of the spinless BTZ black hole with mass $M>0$ "immersed" into $\mathrm{AdS}_{3}$ with length scale $\ell$ is given by

$$
d s^{2}=-\left(\frac{r^{2}-r_{h}^{2}}{\ell^{2}}\right) d t^{2}+\left(\frac{r^{2}-r_{h}^{2}}{\ell^{2}}\right)^{-1} d r^{2}+r^{2} d \phi^{2}
$$

where $t \in]-\infty,+\infty\left[, r>r_{h}\right.$ and $\phi$ has period $2 \pi$. Here $r_{h}=\ell \sqrt{M}$ denotes the horizon radius of the BTZ black hole. Propagating on this gravitational background, we consider a massless minimally coupled scalar field $\Phi$ solution of the wave equation

$$
\square \Phi=0
$$

By inserting the metric (11) into (2), the wave equation provides

$$
\begin{gathered}
-\left(\frac{r^{2}-r_{h}^{2}}{\ell^{2}}\right)^{-1} \frac{\partial^{2} \Phi}{\partial t^{2}}+\left(\frac{r^{2}-r_{h}^{2}}{\ell^{2}}\right) \frac{\partial^{2} \Phi}{\partial r^{2}} \\
+\left(\frac{3 r^{2}-r_{h}^{2}}{r \ell^{2}}\right) \frac{\partial \Phi}{\partial r}+\frac{1}{r^{2}} \frac{\partial^{2} \Phi}{\partial \phi^{2}}=0
\end{gathered}
$$

and we can look for its solutions by separation of variables or, more precisely, by using the ansatz

$$
\Phi_{\nu, \omega}(t, r, \phi)=\frac{1}{\sqrt{r}} f_{\nu, \omega}(r) e^{i(\nu \phi-\omega t)} .
$$

The radial equation satisfied by $f_{\nu, \omega}(r)$ takes the form

$$
\begin{aligned}
& \left(\frac{r^{2}-r_{h}^{2}}{\ell^{2}}\right) \frac{d^{2} f_{\nu, \omega}(r)}{d r^{2}}+\frac{2 r}{\ell^{2}} \frac{d f_{\nu, \omega}(r)}{d r} \\
& \quad+\left[\left(\frac{r^{2}-r_{h}^{2}}{\ell^{2}}\right)^{-1} \omega^{2}-\frac{\nu^{2}}{r^{2}}-\frac{r_{h}^{2}}{4 \ell^{2} r^{2}}-\frac{3}{4 \ell^{2}}\right] f_{\nu, \omega}(r) \\
& \quad=0
\end{aligned}
$$

This differential equation can be solved exactly (see 10 , 11, 12, 13]) in terms of hypergeometric functions [16]. We then obtain for the general form of its solution

$$
\begin{aligned}
& f_{\nu, \omega}(r)=A_{\nu, \omega}\left(\frac{r}{r_{h}}\right)^{\frac{1}{2}}\left(1-\frac{r_{h}^{2}}{r^{2}}\right)^{-i \frac{\ell^{2} \omega}{2 r_{h}}} F\left[-\frac{i \ell}{2 r_{h}}(\ell \omega-\nu),-\frac{i \ell}{2 r_{h}}(\ell \omega+\nu) ; 1-i \frac{\ell^{2} \omega}{r_{h}} ; 1-\frac{r_{h}^{2}}{r^{2}}\right] \\
& \quad+B_{\nu, \omega}\left(\frac{r}{r_{h}}\right)^{\frac{1}{2}}\left(1-\frac{r_{h}^{2}}{r^{2}}\right)^{+i \frac{\ell^{2} \omega}{2 r_{h}}} F\left[+\frac{i \ell}{2 r_{h}}(\ell \omega-\nu),+\frac{i \ell}{2 r_{h}}(\ell \omega+\nu) ; 1+i \frac{\ell^{2} \omega}{r_{h}} ; 1-\frac{r_{h}^{2}}{r^{2}}\right]
\end{aligned}
$$

where $A_{\nu, \omega}$ and $B_{\nu, \omega}$ are arbitrary complex constants.

From now on, we consider more particularly the mode solutions defined by (4) and (6) which correspond to the so-called QNMs and the so-called Regge modes. They are both defined as mode solutions which are purely ingoing at the horizon (i.e., for $r=r_{h}$ ) and vanish at infinity (i.e., for $r=+\infty)$. The first condition selects $B_{\nu, \omega}=0$. By noting that $F(a, b ; c ; 1)=[\Gamma(c) \Gamma(c-a-b)] /[\Gamma(c-$ a) $\Gamma(c-b)]$ if $c \neq 0,-1,-2, \ldots$ and $\operatorname{Re}(c-a-b)>0$, the second one imposes

$$
1-\frac{i \ell}{2 r_{h}}(\ell \omega-\nu)=-n \quad \text { with } \quad n \in \mathbf{N}
$$

or

$$
1-\frac{i \ell}{2 r_{h}}(\ell \omega+\nu)=-n \quad \text { with } \quad n \in \mathbf{N}
$$

Let us first consider the QNMs. Because they are periodic in $\phi$, we must have $\nu=m \in \mathrm{Z}$. They are therefore only defined for the discrete complex values of the frequency $\omega$ (see also Refs. [12, 13])

$$
\omega_{m n}^{ \pm}= \pm \frac{m}{\ell}-i \frac{2 r_{h}}{\ell^{2}}(n+1) \quad \text { with } m \in \mathbf{Z} \text { and } n \in \mathbf{N}
$$

[here, the plus sign correspond to (7a and the minus one to (7b)] and they are given by

$$
\Phi_{m n}^{ \pm}(t, r, \phi)=\frac{1}{\sqrt{r}} f_{m n}^{ \pm}(r) e^{i\left(m \phi-\omega_{m n}^{ \pm} t\right)}
$$

with

$$
\begin{aligned}
& f_{m n}^{ \pm}(r)=A_{m n}^{ \pm}\left(\frac{r}{r_{h}}\right)^{\frac{1}{2}}\left(1-\frac{r_{h}^{2}}{r^{2}}\right)^{-i \frac{\ell^{2} \omega_{m n}^{ \pm}}{2 r_{h}}} \\
& \times F\left[-n-1,-\frac{i \ell}{2 r_{h}}\left(\ell \omega_{m n}^{ \pm} \pm m\right) ; 1-i \frac{\ell^{2} \omega_{m n}^{ \pm}}{r_{h}} ; 1-\frac{r_{h}^{2}}{r^{2}}\right] .
\end{aligned}
$$


It should be noted that $\forall m \in \mathbf{Z}, \omega_{-m n}^{+}=\omega_{m n}^{-}$but $\Phi_{-m n}^{+}(t, r, \phi) \neq \Phi_{m n}^{-}(t, r, \phi)$. As a consequence, each QNM complex frequency is two-fold degenerated.

Let us now consider the Regge modes. They are defined on the covering space of the BTZ black hole obtained by relaxing the condition of periodicity in the coordinate $\phi$, i.e., by considering that $\phi \in]-\infty,+\infty[$, and by furthermore assuming that $\omega>0$. The covering space of the BTZ black hole considered here is, in fact, nothing other than one of the 12 charts which permits us to provide a global covering of $\mathrm{AdS}_{3}$ in BTZ coordinates [17]. However, it should be noted that we do not work on $\mathrm{AdS}_{3}$ : relaxing the condition of periodicity in the coordinate $\phi$ is just a trick which will permit us to consider multivalued mode solutions of the wave equation (2) which describe surface waves propagating around the BTZ black hole and to take into account their multiple circumnavigations as well as the associated radiation damping due to their attenuations. Such a trick has been invented by Sommerfeld in order to analyze scattering by spheres and to emphasize the role of surface waves (see Ref. 14] and, more particularly, Appendix II of Chapter $\mathrm{V}$ as well as the appendix of Chapter VI). The Sommerfeld approach is an alternative to the usual CAM approach of scattering developed by Watson [15]. It allows us to use the Regge pole concept in a framework where the notion of an $S$ matrix does not exist, and therefore to extend our CAM analysis of the Schwarzschild black hole [1] to the BTZ one.

The Regge modes of the BTZ black hole are only defined for particular complex values of the parameter $\nu$ which we shall call Regge poles even if they are not the poles of an $S$ matrix. These Regge poles are exactly given by

$$
\nu_{n}^{ \pm}(\omega)= \pm \omega \ell \pm i \frac{2 r_{h}}{\ell}(n+1) \quad \text { with } \omega>0 \text { and } n \in \mathbf{N}
$$

[here, the plus sign correspond to (7a) and the minus one to $(7 \mathrm{~b})]$ and the corresponding Regge modes are given by

$$
\begin{aligned}
\Phi_{\nu_{n}(\omega)}^{ \pm}(t, r, \phi) & =\frac{1}{\sqrt{r}} f_{\nu_{n}(\omega)}^{ \pm}(r) e^{i\left[\nu_{n}^{ \pm}(\omega) \phi-\omega t\right]} \\
& =\frac{1}{\sqrt{r}} f_{\nu_{n}(\omega)}^{ \pm}(r) e^{\mp \frac{2 r_{h}}{\ell}(n+1) \phi} e^{i[ \pm \omega \ell \phi-\omega t]}
\end{aligned}
$$

with

$$
\begin{aligned}
& f_{\nu_{n}(\omega)}^{ \pm}(r)=A_{\nu_{n}(\omega)}^{ \pm}\left(\frac{r}{r_{h}}\right)^{\frac{1}{2}}\left(1-\frac{r_{h}^{2}}{r^{2}}\right)^{-i \frac{\ell^{2} \omega}{2 r_{h}}} \\
& \times F\left[-n-1, n+1-i \frac{\ell^{2} \omega}{r_{h}} ; 1-i \frac{\ell^{2} \omega}{r_{h}} ; 1-\frac{r_{h}^{2}}{r^{2}}\right] .
\end{aligned}
$$

It should be noted that, even if the radial parts of $\Phi_{\nu_{n}(\omega)}^{+}$ and $\Phi_{\nu_{n}(\omega)}^{-}$are identical, these mode solutions are different. In fact, because we have $\nu_{n}^{+}(\omega)=-\nu_{n}^{-}(\omega)$, they respectively describe waves with identical properties but propagating counterclockwise and clockwise with an exponential decay around the BTZ black hole, with $\left|\operatorname{Re} \nu_{n}^{ \pm}(\omega)\right|=\omega \ell$ representing their azimuthal propagation constant and $\left|\operatorname{Im} \nu_{n}^{ \pm}(\omega)\right|=\left(2 r_{h} / \ell\right)(n+1)$ their damping constant.

\section{B. More on the physical interpretation of the Regge modes}

The Regge poles and the Regge modes obtained in the previous subsection can be semiclassically interpreted in terms of surface waves supported by the boundary at infinity of the BTZ black hole, this boundary furthermore playing the role of a photon sphere. We shall now discuss more precisely these two important results which permit us to establish some interesting analogies between the BTZ and the Schwarzschild black holes.

The propagative behavior in $\exp \left(i\left[\operatorname{Re} \nu_{n}^{ \pm}(\omega) \phi-\omega t\right]\right)=$ $\exp (i[ \pm \omega \ell \phi-\omega t])$ of the Regge modes (12)-(13) permits us to note that the waves they describe circle the BTZ black hole in time

$$
T=\frac{2 \pi}{\omega}\left|\operatorname{Re} \nu_{n}^{ \pm}(\omega)\right|=2 \pi \ell .
$$

Furthermore, a scalar photon (associated with the massless scalar field $\Phi)$ on the circular orbit with constant radius $R$ takes the time

$$
T^{\prime}=\frac{2 \pi R}{\sqrt{\frac{R^{2}-r_{h}^{2}}{\ell^{2}}}}=\frac{2 \pi R}{\sqrt{\frac{R^{2}}{\ell^{2}}-M}}
$$

to circle the BTZ black hole. Such result can be easily found by solving $d s^{2}=0$ with $d s^{2}$ given by (1), i.e. by integrating the equation of a circular null geodesic. By equating $T$ and $T^{\prime}$, we obtain that necessarily $R \rightarrow+\infty$. In other words, the circular orbit of the scalar photon lies on the BTZ black hole boundary and we can consider that the set of Regge modes constitutes a family indexed by $n \in \mathbf{N}$ of surface waves supported by this boundary.

Let us now consider the photon sphere of the BTZ black hole. We recall that, for a static spherically symmetric black hole of dimension $d$ with metric of the form

$$
d s^{2}=-g_{t t}(r) d t^{2}+g_{r r}(r) d r^{2}+r^{2} d \Omega_{d-2}^{2},
$$

the photon sphere is defined by the greater positive solution of the equation

$$
\frac{g_{t t}^{\prime}(r)}{g_{t t}(r)}=\frac{2}{r}
$$

[see Ref. [18] for a general definition and an extension of the photon sphere concept in an arbitrary space-time and Eq. (54) of this paper]. For the BTZ black hole, (17) admits a unique formal solution for $r \rightarrow+\infty$. In that sense, the boundary of the BTZ black hole can be formally considered as its photon sphere which then acts as the support of the Regge surface waves (12)-(13). 


\section{DIFFRACTED FEYNMAN PROPAGATOR AND QUASINORMAL FREQUENCIES}

The Feynman propagator associated with the scalar field $\Phi$ as well as the corresponding retarded and advanced Green functions satisfy the wave equation

$$
\square_{x} G\left(x, x^{\prime}\right)=-\delta^{3}\left(x, x^{\prime}\right)
$$

which, in the BTZ black hole space-time defined by (1), takes the form

$$
\begin{gathered}
{\left[-\left(\frac{r^{2}-r_{h}^{2}}{\ell^{2}}\right)^{-1} \frac{\partial^{2}}{\partial t^{2}}+\left(\frac{r^{2}-r_{h}^{2}}{\ell^{2}}\right) \frac{\partial^{2}}{\partial r^{2}}\right.} \\
\left.+\left(\frac{3 r^{2}-r_{h}^{2}}{r \ell^{2}}\right) \frac{\partial}{\partial r}+\frac{1}{r^{2}} \frac{\partial^{2}}{\partial \phi^{2}}\right] G\left(t, r, \phi ; t^{\prime}, r^{\prime}, \phi^{\prime}\right) \\
\quad=-\frac{1}{r} \delta\left(t-t^{\prime}\right) \delta\left(r-r^{\prime}\right) \delta\left(\phi-\phi^{\prime}\right) .
\end{gathered}
$$

All these Green functions can be constructed by Fourier transform from the Green function $G_{\omega}\left(r, \phi ; r^{\prime}, \phi^{\prime}\right)$ with $\omega>0$ defined as the symmetric solution of the Helmholtz-type equation

$$
\begin{aligned}
{\left[\left(\frac{r^{2}-r_{h}^{2}}{\ell^{2}}\right) \frac{\partial^{2}}{\partial r^{2}}\right.} & +\left(\frac{3 r^{2}-r_{h}^{2}}{r \ell^{2}}\right) \frac{\partial}{\partial r} \\
+\frac{1}{r^{2}} \frac{\partial^{2}}{\partial \phi^{2}} & \left.+\left(\frac{r^{2}-r_{h}^{2}}{\ell^{2}}\right)^{-1} \omega^{2}\right] G_{\omega}\left(r, \phi ; r^{\prime}, \phi^{\prime}\right) \\
& =-\frac{1}{2 \pi r} \delta\left(r-r^{\prime}\right) \delta\left(\phi-\phi^{\prime}\right)
\end{aligned}
$$

which vanishes at infinity. For example, we have for the Feynman propagator

$$
\begin{gathered}
G_{F}\left(t, r, \phi ; t^{\prime}, r^{\prime}, \phi^{\prime}\right)=\frac{1}{2 \pi} \int_{-\infty}^{+\infty}\left[\Theta(+\omega) G_{+\omega}\left(r, \phi ; r^{\prime}, \phi^{\prime}\right)\right. \\
\left.+\Theta(-\omega) G_{-\omega}\left(r, \phi ; r^{\prime}, \phi^{\prime}\right)\right] e^{i\left[\omega\left(t-t^{\prime}\right)\right]} d \omega .
\end{gathered}
$$

Here, $\Theta$ denotes the Heaviside step function and it should be noted that, in order to construct the Feynman propagator $G_{F}\left(t, r, \phi ; t^{\prime}, r^{\prime}, \phi^{\prime}\right)$, we need the Green function $G_{\omega}\left(r, \phi ; r^{\prime}, \phi^{\prime}\right)$ also for $\omega<0$. In fact, as we shall see later, we will also need $G_{\omega}\left(r, \phi ; r^{\prime}, \phi^{\prime}\right)$ in the full complex $\omega$-plane in order to discuss the resonant aspects of the BTZ black hole. It can be obtained by analytic continuation from its expression for $\omega>0$.

From now on, we shall mainly focus our attention on the Green function $G_{\omega}\left(r, \phi ; r^{\prime}, \phi^{\prime}\right)$. Following Sommerfeld [14] (see also Sec. 2.1 and Appendix A.1 of Ref. [19] for a pedagogical introduction to the Sommerfeld method and the full paper for its application to cylinders), we construct $G_{\omega}\left(r, \phi ; r^{\prime}, \phi^{\prime}\right)$ from the Regge modes defined in the previous section or, more precisely, we seek it in the form

$$
\begin{gathered}
G_{\omega}^{d}\left(r, \phi ; r^{\prime}, \phi^{\prime}\right)=\frac{1}{\sqrt{r}} \sum_{n=0}^{+\infty}\left[f_{\nu_{n}^{+}(\omega)}(r) V_{n, \omega}^{+}\left(\phi ; r^{\prime}, \phi^{\prime}\right)\right. \\
\left.\quad+f_{\nu_{n}^{-}(\omega)}(r) V_{n, \omega}^{-}\left(\phi ; r^{\prime}, \phi^{\prime}\right)\right] .
\end{gathered}
$$

Here we consider that the functions $f_{\nu_{n}^{ \pm}(\omega)}(r)$ are given by (13) with $A_{\nu_{n}(\omega)}^{ \pm}=1$ and we assume that the functions $V_{n, \omega}^{ \pm}\left(\phi ; r^{\prime}, \phi^{\prime}\right)$ are such that $G_{\omega}^{d}\left(r, \phi ; r^{\prime}, \phi^{\prime}\right)$ is a solution of (20) symmetric under the exchange $(r, \phi) \leftrightarrow\left(r^{\prime}, \phi^{\prime}\right)$. Furthermore, by working in Sec. II with the Regge modes, we have deferred the imposition of periodicity in the coordinate $\phi$ on the solutions of the wave equation but now we shall impose this condition on (22) and therefore on (21).

It is important to note that the Green function constructed by inserting (22) into (21) differs from the Feynman propagator which could be obtained from the exact normalized mode solutions of the wave equation (2). Indeed, the Regge modes do not constitute a complete system of solution of this equation. In fact, the Sommerfeld method permits us to only consider that part of the exact Feynman propagator which describe more particularly "diffraction" by the BTZ black hole. Such a result was noted by Sommerfeld for its analysis of scattering by spheres and remains valid in the present context. This drawback is not too serious because, in fact, it is the diffractive part of the Feynman propagator which contains all the information about the resonant aspects of the problem.

By inserting (22) into (20) and by using (5) with $\nu=\nu_{n}^{ \pm}(\omega)$, we find that the functions $V_{n, \omega}^{ \pm}\left(\phi ; r^{\prime}, \phi^{\prime}\right)$ must satisfy

$$
\begin{gathered}
\frac{1}{r^{2}} \sum_{n=0}^{+\infty}\left[f_{\nu_{n}^{+}(\omega)}(r)\left(\frac{\partial^{2}}{\partial \phi^{2}}+\nu_{n}^{+}(\omega)^{2}\right) V_{n, \omega}^{+}\left(\phi ; r^{\prime}, \phi^{\prime}\right)\right. \\
\left.+f_{\nu_{n}^{-}(\omega)}(r)\left(\frac{\partial^{2}}{\partial \phi^{2}}+\nu_{n}^{-}(\omega)^{2}\right) V_{n, \omega}^{-}\left(\phi ; r^{\prime}, \phi^{\prime}\right)\right] \\
=-\frac{1}{2 \pi \sqrt{r}} \delta\left(r-r^{\prime}\right) \delta\left(\phi-\phi^{\prime}\right) .
\end{gathered}
$$

We then multiply (23) by $f_{\nu_{p}^{ \pm}(\omega)}(r)$ and integrate over the radial domain $r \in\left[r_{h},+\infty\right.$ [ which contains $r^{\prime}$. The orthonormalization relation

$$
\int_{r_{h}}^{+\infty} \frac{1}{r^{2}} f_{\nu_{n}^{ \pm}(\omega)}(r) f_{\nu_{p}^{ \pm}(\omega)}(r) d r= \pm N_{n}^{ \pm}(\omega) \delta_{n p}
$$

with

$$
\begin{aligned}
N_{n}^{ \pm}(\omega) & =i \frac{n !(n+1) !}{2 \ell \nu_{n}^{ \pm}(\omega)} \\
& \times \frac{\left[\Gamma\left(1-i \frac{\ell^{2} \omega}{r_{h}}\right)\right]^{2}}{\Gamma\left(n+1-i \frac{\ell^{2} \omega}{r_{h}}\right) \Gamma\left(n+2-i \frac{\ell^{2} \omega}{r_{h}}\right)}
\end{aligned}
$$

for the Regge radial modes which can be obtained from (5) by generalizing, mutatis mutandis, the calculation displayed in Appendix A.1 of Ref. [19], permits us to write

$$
\begin{gathered}
\left(\frac{\partial^{2}}{\partial \phi^{2}}+\nu_{n}^{ \pm}(\omega)^{2}\right) V_{n, \omega}^{ \pm}\left(\phi ; r^{\prime}, \phi^{\prime}\right)= \\
\mp \frac{1}{2 \pi N_{n}^{ \pm}(\omega)} \frac{f_{\nu_{n}^{ \pm}(\omega)}\left(r^{\prime}\right)}{\sqrt{r^{\prime}}} \delta\left(\phi-\phi^{\prime}\right) .
\end{gathered}
$$


The general solution of (26) can be sought in the form

$$
\begin{array}{r}
V_{n, \omega}^{ \pm}\left(\phi ; r^{\prime}, \phi^{\prime}\right)=\Theta\left(\phi^{\prime}-\phi\right)\left[\mathcal{A}_{n, \omega}^{ \pm}\left(r^{\prime}, \phi^{\prime}\right) \cos \left(\nu_{n}^{ \pm}(\omega) \phi\right)\right. \\
\left.+\mathcal{B}_{n, \omega}^{ \pm}\left(r^{\prime}, \phi^{\prime}\right) \sin \left(\nu_{n}^{ \pm}(\omega) \phi\right)\right] \\
\quad+\Theta\left(\phi-\phi^{\prime}\right)\left[\mathcal{C}_{n, \omega}^{ \pm}\left(r^{\prime}, \phi^{\prime}\right) \cos \left(\nu_{n}^{ \pm}(\omega) \phi\right)\right. \\
\left.+\mathcal{D}_{n, \omega}^{ \pm}\left(r^{\prime}, \phi^{\prime}\right) \sin \left(\nu_{n}^{ \pm}(\omega) \phi\right)\right] .
\end{array}
$$

Then, by inserting (27) into (26) and by using the condition that the functions $V_{n, \omega}^{ \pm}\left(\phi ; r^{\prime}, \phi^{\prime}\right)$ and their derivatives must be single-valued (now, we impose periodicity in the coordinate $\phi)$, we determine the functions $\mathcal{A}_{n, \omega}^{ \pm}\left(r^{\prime}, \phi^{\prime}\right)$,
$\mathcal{B}_{n, \omega}^{ \pm}\left(r^{\prime}, \phi^{\prime}\right), \mathcal{C}_{n, \omega}^{ \pm}\left(r^{\prime}, \phi^{\prime}\right)$ and $\mathcal{D}_{n, \omega}^{ \pm}\left(r^{\prime}, \phi^{\prime}\right)$ and we obtain

$$
\begin{aligned}
& V_{n, \omega}^{ \pm}\left(\phi ; r^{\prime}, \phi^{\prime}\right)=\mp \frac{f_{\nu_{n}^{ \pm}(\omega)}\left(r^{\prime}\right) / \sqrt{r^{\prime}}}{4 \pi N_{n}^{ \pm}(\omega) \nu_{n}^{ \pm}(\omega) \sin \left[\pi \nu_{n}^{ \pm}(\omega)\right]} \\
& \times\left[\Theta\left(\phi^{\prime}-\phi\right) \cos \left[\nu_{n}^{ \pm}(\omega)\left(\pi-\phi^{\prime}+\phi\right)\right]\right. \\
& \left.+\Theta\left(\phi-\phi^{\prime}\right) \cos \left[\nu_{n}^{ \pm}(\omega)\left(\pi+\phi^{\prime}-\phi\right)\right]\right] .
\end{aligned}
$$

Now, by inserting (28) into (22) and by taking into account the expression (25) of the normalization factor $N_{n}^{ \pm}(\omega)$, we obtain the final result

$$
\begin{array}{r}
G_{\omega}^{d}\left(r, \phi ; r^{\prime}, \phi^{\prime}\right)=+\frac{i \ell}{2 \pi} \sum_{n=0}^{+\infty} \frac{a_{n}(\omega)}{\sin \left[\pi \nu_{n}^{+}(\omega)\right]} \frac{f_{\nu_{n}^{+}(\omega)}(r) f_{\nu_{n}^{+}(\omega)}\left(r^{\prime}\right)}{\sqrt{r r^{\prime}}}\left\{\Theta\left(\phi^{\prime}-\phi\right) \cos \left[\nu_{n}^{+}(\omega)\left(\pi-\phi^{\prime}+\phi\right)\right]\right. \\
\left.+\Theta\left(\phi-\phi^{\prime}\right) \cos \left[\nu_{n}^{+}(\omega)\left(\pi-\phi+\phi^{\prime}\right)\right]\right\} \\
-\frac{i \ell}{2 \pi} \sum_{n=0}^{+\infty} \frac{a_{n}(\omega)}{\sin \left[\pi \nu_{n}^{-}(\omega)\right]} \frac{f_{\nu_{n}^{-}(\omega)}(r) f_{\nu_{n}^{-}(\omega)}\left(r^{\prime}\right)}{\sqrt{r r^{\prime}}}\left\{\Theta\left(\phi-\phi^{\prime}\right) \cos \left[\nu_{n}^{-}(\omega)\left(\pi-\phi+\phi^{\prime}\right)\right]\right. \\
\left.+\Theta\left(\phi^{\prime}-\phi\right) \cos \left[\nu_{n}^{-}(\omega)\left(\pi-\phi^{\prime}+\phi\right)\right]\right\}
\end{array}
$$

with

$$
a_{n}(\omega)=\frac{\Gamma\left(n+1-i \frac{\ell^{2} \omega}{r_{h}}\right) \Gamma\left(n+2-i \frac{\ell^{2} \omega}{r_{h}}\right)}{n !(n+1) !\left[\Gamma\left(1-i \frac{\ell^{2} \omega}{r_{h}}\right)\right]^{2}}=\frac{\left(n+1-i \frac{\ell^{2} \omega}{r_{h}}\right)\left[\left(1-i \frac{\ell^{2} \omega}{r_{h}}\right)_{n}\right]^{2}}{n !(n+1) !} .
$$

Here the symmetry of $G_{\omega}^{d}\left(r, \phi ; r^{\prime}, \phi^{\prime}\right)$ under the exchange $(r, \phi) \leftrightarrow\left(r^{\prime}, \phi^{\prime}\right)$ appears explicitly. $G_{\omega}^{d}\left(r, \phi ; r^{\prime}, \phi^{\prime}\right)$ has been constructed for $\omega>0$. By analytic continuation from the positive real $\omega$-axis, it is obtained in the full complex $\omega$-plane.

Before exploiting the expression of $G_{\omega}^{d}\left(r, \phi ; r^{\prime}, \phi^{\prime}\right)$ given by (29) and (30) in order to recover the resonant aspects of the BTZ black hole, it seems to us interesting to make a digression which provide a physical interpretation of $G_{\omega}^{d}\left(r, \phi ; r^{\prime}, \phi^{\prime}\right)$. By using

$$
\frac{1}{\sin \pi \nu}=-2 i \sum_{k=0}^{+\infty} e^{+i \pi(2 k+1) \nu} \quad \text { for } \quad \operatorname{Im} \nu>0 \quad \text { and } \quad \frac{1}{\sin \pi \nu}=+2 i \sum_{k=0}^{+\infty} e^{-i \pi(2 k+1) \nu} \quad \text { for } \quad \operatorname{Im} \nu<0,
$$

we can write

$$
\begin{aligned}
& G_{\omega}^{d}\left(r, \phi ; r^{\prime}, \phi^{\prime}\right)=+\frac{\ell}{2 \pi} \sum_{n=0}^{+\infty} a_{n}(\omega) \frac{f_{\nu_{n}^{+}(\omega)}(r) f_{\nu_{n}^{+}(\omega)}\left(r^{\prime}\right)}{\sqrt{r r^{\prime}}} \\
& \times\left\{\Theta\left(\phi^{\prime}-\phi\right)\left[\sum_{k=0}^{+\infty} e^{i\left[\nu_{n}^{+}(\omega)\left(\phi^{\prime}-\phi+k 2 \pi\right)\right]}+e^{i\left[\nu_{n}^{+}(\omega)\left(2 \pi-\phi^{\prime}+\phi+k 2 \pi\right)\right]}\right]+\phi \leftrightarrow \phi^{\prime}\right\} \\
&+\frac{\ell}{2 \pi} \sum_{n=0}^{+\infty} a_{n}(\omega) \frac{f_{\nu_{n}^{-}(\omega)}(r) f_{\nu_{n}^{-}(\omega)}\left(r^{\prime}\right)}{\sqrt{r r^{\prime}}} \\
& \times\left\{\Theta\left(\phi-\phi^{\prime}\right)\left[\sum_{k=0}^{+\infty} e^{-i\left[\nu_{n}^{-}(\omega)\left(\phi-\phi^{\prime}+k 2 \pi\right)\right]}+e^{-i\left[\nu_{n}^{-}(\omega)\left(2 \pi-\phi+\phi^{\prime}+k 2 \pi\right)\right]}\right]+\phi^{\prime} \leftrightarrow \phi\right\} .
\end{aligned}
$$

By reinstating the temporal dependance in $e^{i \omega\left(t-t^{\prime}\right)}$ into Eq. (32) [see Eq. (21)], this expression provides a physi- 
cal interpretation of the diffractive Feynman propagator. It appears as a sum over $n \in \mathbf{N}$, i.e., over all the surface waves supported by the BTZ black hole boundary. In that sum, terms like $e^{i\left[\nu_{n}^{ \pm}(\omega)\left(\phi^{\prime}-\phi+k 2 \pi\right)\right]}$ correspond to the contributions of surface waves propagating around the black hole and the sums over the index $k$ take into account their multiple circumnavigations.

We shall now complete this section by considering the analytic structure of $G_{\omega}^{d}\left(r, \phi ; r^{\prime}, \phi^{\prime}\right)$ in the complex $\omega$ plane and, more precisely, by looking for its poles in this plane because they correspond to the resonance frequencies of the scalar field $\Phi$ propagating in the BTZ black hole space-time. The only poles of $G_{\omega}^{d}\left(r, \phi ; r^{\prime}, \phi^{\prime}\right)$ are the zeros of the functions $\sin \left[\pi \nu_{n}^{ \pm}(\omega)\right]$ with $n \in \mathbf{N}$. They are therefore obtained by solving

$$
\nu_{n}^{ \pm}(\omega)=m \quad \text { with } m \in \mathbf{Z}
$$

which provides the exact results given by (8) for the QNM complex frequencies and which furthermore clarifies the meaning of the indices $n$ and $m$ introduced to denote them: the QNM complex frequencies are grouped into families labeled by the indices $n \in \mathbf{N}$, each family being associated with a given surface wave or equivalently with a Regge pole, and the members of a given family are indexed by $m \in \mathbf{Z}$.

It is worth noting that the method we have developed in this article should not only be regarded as a new way to calculate the QNM complex frequencies $\omega_{m n}^{ \pm}$. It is, above all, an approach which permits us to physically interpret them: they appear as Breit-Wigner-type resonances generated by the family of surface waves supported by the BTZ black hole boundary at infinity. Indeed:

- In the immediate neighborhood of a QNM complex frequency $\omega_{m n}^{ \pm}, G_{\omega}^{d}\left(r, \phi ; r^{\prime}, \phi^{\prime}\right)$ given by (29) has the Breit-Wigner form, i.e., we can write

$$
G_{\omega}^{d}\left(r, \phi ; r^{\prime}, \phi^{\prime}\right) \approx \frac{\mathcal{N}_{\omega}^{ \pm}\left(r, \phi ; r^{\prime}, \phi^{\prime}\right)}{\omega-\omega_{m n}^{ \pm(o)}+i \Gamma_{m n}^{ \pm} / 2} .
$$

with $\omega_{m n}^{ \pm(o)}= \pm m / \ell$ and $\Gamma_{m n}^{ \pm} / 2=\left(2 r_{h} / \ell^{2}\right)(n+1)$. This result is a direct consequence of the formula $\sin (\pi x) \approx$ $(-1)^{m} \pi(x-m)$ for $x \rightarrow m$ with $m \in \mathbf{Z}$.

- For a given value of $n$, a term like $1 / \sin \left[\pi \nu_{n}^{ \pm}(\omega)\right]$ is produced by interference between the different components of the $n$-th Regge surface wave supported by the black hole boundary [see Eq. (31) and compare (29) with (32)], each component corresponding to a different number of circumnavigations. Furthermore, a constructive interference between its different components occurs when the quantity $\operatorname{Re} \nu_{n}^{ \pm}(\omega)$ coincides with an integer, i.e., for real resonance frequencies $\omega_{m n}^{ \pm(o)}$ obtained from the Bohr-Sommerfeld-type quantization conditions

$$
\operatorname{Re} \nu_{n}^{ \pm}\left(\omega_{m n}^{ \pm(o)}\right)=m \quad m \in \mathbf{Z} .
$$

This equation provides again the real parts $\omega_{m n}^{ \pm(o)}$ of the family of QNM complex frequencies $\omega_{m n}^{ \pm}$generated by the $n$-th Regge surface wave.

\section{CONCLUSION AND PERSPECTIVES}

In the present paper, by considering the Regge poles of the spinless BTZ black hole, we have provided a new interpretation for its QNMs: they can be considered as generated by surface waves propagating on its boundary and the associated complex frequencies are Breit-Wignertype resonances. This interpretation can be easily extended to the rotating BTZ black hole [20]. In that case, the Regge modes are associated with the Regge poles

$$
\begin{aligned}
& \nu_{n}^{+}(\omega)=+\omega \ell+i \frac{2\left(r_{+}-r_{-}\right)}{\ell}(n+1) \\
& \nu_{n}^{-}(\omega)=-\omega \ell-i \frac{2\left(r_{+}+r_{-}\right)}{\ell}(n+1)
\end{aligned}
$$

with $\omega>0$ and $n \in \mathbf{N}$. Here $r_{+}$and $r_{-}$denote the outer and inner horizon radii of the rotating BTZ black hole which are linked with its mass $M$ and its angular momentum $J$ by $M=\left(r_{+}^{2}+r_{-}^{2}\right) / \ell^{2}$ and $J=2 r_{+} r_{-} / \ell$. The Regge modes correspond again to surface waves supported by the boundary of the black hole but, now, it should be noted that they describe surface waves with different properties. Indeed, even if they have the same azimuthal propagation constant $\left|\operatorname{Re} \nu_{n}^{ \pm}(\omega)\right|=\omega \ell$, their attenuations are different: the damping constant $\left|\operatorname{Im} \nu_{n}^{+}(\omega)\right|=\left[2\left(r_{+}-r_{-}\right) / \ell\right](n+1)$ of the wave which propagates counterclockwise around the BTZ black hole (i.e., which is in co-rotation with the black hole) is lesser than the damping constant $\left|\operatorname{Im} \nu_{n}^{-}(\omega)\right|=\left[2\left(r_{+}+\right.\right.$ $\left.\left.r_{-}\right) / \ell\right](n+1)$ of the wave which propagates clockwise. Such a behavior leads directly to the splitting of the QNM complex frequencies: when we insert (36a) and (36b) into the resonance condition (33), we obtain the exact results [13]

$$
\begin{aligned}
& \omega_{m n}^{+}=+\frac{m}{\ell}-i \frac{2\left(r_{+}-r_{-}\right)}{\ell^{2}}(n+1) \\
& \omega_{m n}^{-}=-\frac{m}{\ell}-i \frac{2\left(r_{+}+r_{-}\right)}{\ell^{2}}(n+1)
\end{aligned}
$$

with $m \in \mathbf{Z}$ and $n \in \mathbf{N}$. Now we have $\omega_{-m n}^{+} \neq \omega_{m n}^{-}$ and the two-fold degeneracy of the QNM complex frequencies noted in Sec. II is removed due to the rotation of the BTZ black hole which induces different damping for the surface waves propagating counterclockwise and clockwise.

Because the BTZ black hole geometry frequently appears as a factor in the near horizon geometry of higher dimensional black holes of string theories (see, e.g., Ref. 21]), our Regge poles analysis could be directly generalized and analogous results could be obtained in a more general context. Similarly, it would be interesting to explore the more general situation of quantum corrected BTZ black holes (see, e.g., Ref. [22]). We believe that all these results could be helpful in order to shed light on AdS/CFT correspondence from a new point of view. Unfortunately, we have been unable to make important steps in this direction. In particular, we do not 
actually have at our disposal a clear $\mathrm{CFT}_{2}$-interpretation of the BTZ Regge poles analogous to the interpretation of the QNM complex frequencies provided in Refs. [23, 24].

It is finally important to recall that the boundary of the BTZ black hole can also be considered as its photon sphere. It is therefore quite tempting to wonder if the photon sphere of all the other black holes might play a central role in the context of holography. In particular, could one holographically map a quantum field theory (or a string theory) defined on the Schwarzschild black hole of mass $M$ on a conformally invariant quantum field theory defined on its photon sphere at $r=3 M$ ? And more generally, could one not systematically consider photon spheres as holographic screens?

\section{Acknowledgments}

We thank Stéphane Ancey, Denis Bernard, Paul Gabrielli, Bruce Jensen and Bernard Raffaelli for various discussions concerning some of the topics considered in this article.
[1] Y. Décanini, A. Folacci, and B. P. Jensen, Phys. Rev. D 67, 124017 (2003).

[2] N. Andersson and K.-E. Thylwe, Class. Quantum Grav. 11, 2991 (1994).

[3] N. Andersson, Class. Quantum Grav. 11, 3003 (1994).

[4] C. J. Goebel, Ap. J. 172, L95 (1972).

[5] V. Ferrari and B. Mashhoon, Phys. Rev. D 30, 295 (1984).

[6] B. Mashhoon, Phys. Rev. D 31, 290 (1985).

[7] J. M. Stewart, Proc. R. Soc. Lond. A 424, 239 (1989).

[8] N. Andersson and H. Onozawa, Phys. Rev. D 54, 7470 (1996).

[9] M. Bañados, C. Teitelboim, and J. Zanelli, Phys. Rev. Lett. 69, 1849 (1992).

[10] K. Ghoroku and A. L. Larsen, Phys. Lett. B 328, 28 (1994).

[11] I. Ichinose and Y. Satoh, Nucl. Phys. B 447, 340 (1995).

[12] V. Cardoso and J. P. S. Lemos, Phys. Rev. D 63, 124015 (2001).

[13] D. Birmingham, Phys. Rev. D 64, 064024 (2001).
[14] A. Sommerfeld, Partial Differential Equations of Physics (Academic Press, New York, 1949).

[15] G. N. Watson, Proc. R. Soc. London A 95, 83 (1918).

[16] M. Abramowitz and I. A. Stegun, Handbook of Mathematical Functions (Dover, New-York, 1965).

[17] M. Bañados, M. Henneaux, C. Teitelboim, and J. Zanelli, Phys. Rev. D 48, 1506 (1993).

[18] C.-M. Claudel, K. S. Virbhadra, and G. F. R. Ellis, J. Math. Phys. 42, 818 (2001).

[19] S. Ancey, A. Folacci, and P. Gabrielli, J. Phys. A: Math. Gen. 33, 3179 (2000).

[20] Y. Decanini and A. Folacci (unpublished notes).

[21] O. Aharony, S. S. Gubser, J. Maldacena, H. Ooguri, and Y. Oz, Phys. Rep. 323, 183 (2000).

[22] R. A. Konoplya, Phys. Rev. D 70, 047503 (2004).

[23] D. Birmingham, I. Sachs, and S. N. Solodukhin, Phys. Rev. Lett. 88, 151301 (2002).

[24] D. Birmingham, I. Sachs, and S. N. Solodukhin, Phys. Rev. D 67, 104026 (2003). 